\title{
Solidification of a Supercooled Liquid in a Narrow Channel
}

\author{
Mohsen Sabouri-Ghomi, ${ }^{1}$ Nikolas Provatas, ${ }^{1,2}$ Martin Grant ${ }^{1}$ \\ ${ }^{1}$ Physics Department and Centre for the Physics of Materials, McGill University, Rutherford Building, 3600 rue University, \\ Montréal, Québec, Canada H3A $2 T 8$ \\ ${ }^{2}$ Pulp and Paper Research Institute of Canada, 570 Boulevard St.-Jean, Pointe-Claire, Québec, Canada, H9R 3J9
}

(Received 5 December 2000)

\begin{abstract}
We simulate solidification in a narrow channel through the use of a phase-field model with an adaptive grid. In different regimes, we find that the solid can grow in fingerlike steady-state shapes, or become unstable, exhibiting unsteady growth. At low melt undercoolings, we find good agreement between our results, theoretical predictions, and experiment. For high undercoolings, we report evidence for a new stable steady-state finger shape which exists in experimentally accessible ranges for typical materials.
\end{abstract}

PACS numbers: 64.60.My, 05.70.Ln

The classic example of pattern selection in driven systems is the shape of a dendritic crystal's tip, which is growing into a supercooled melt, mediated by the diffusion of the latent heat of solidification [1,2]. Simple theories, which neglect the effect of surface tension $\kappa$, predict that only the product of the velocity $V$ and dendrite tip radius $\rho$ is determined for a given undercooling. However, the modern theory of microscopic solvability, which incorporates the surface tension, predicts the unique operating condition for a steady-state tip, provided that $\kappa$ is anisotropic [2]. This work generalized earlier work on local models of solidification [3,4] and, in particular, work on the closely related problem of viscous fingering in Hele-Shaw cells [5]. The latter phenomena correspond to the fingering instability which occurs when a relatively inviscid fluid, such as water, is forced by a pressure gradient to invade a more viscous fluid, such as oil. It can be shown that, if this process takes place between two glass plates which form a long channel of width $L$-called a Hele-Shaw cell-a long steady-state finger forms and grows [6]. As in the case for dendritic tips, in the absence of surface tension, theory predicts fingers of any width. The theory of microscopic solvability predicts a unique finger width, e.g., in units of the channel width, the finger width approaches $\lambda=1 / 2$ in the limit of $\kappa \rightarrow 0$.

Mathematically, both the dendrite tip and the viscous finger are described by similar equations, namely, diffusion or the Laplace problem in the bulk phases, subject to the boundary conditions of the moving interface, and the driving force at infinity far from the interface. In the limit in which the diffusion length $l_{D}$ becomes very large compared to the channel width, where $l_{D}=D / V$ and $D$ is the diffusion coefficient (that is, if the Péclet number $P=L / l_{D}$ is small), the two problems become almost identical. This close relationship has led to many beautiful insights into both phenomena [7-9]. For example, the "free" viscous finger generically shows unsteady tip splitting behavior, which can be attributed to the fluid's isotropic surface tension. However, creating an effective anisotropic surface tension, by scratching one of the glass plates, stabilizes the tip, giving rise to dendritic patterns [9].

In this Letter, we report the results of a theoretical investigation into the alternate case: solidification constrained to grow in two dimensions within a long channel. For small $P$, this is closely related to viscous fingering with anisotropic surface tension. We compare our numerical results for a phase-field model of solidification, with no adjustable parameters, to theory [7] and experiment [8], finding good agreement, thereby providing the first quantitative test of solvability theory in this context. For larger $P$, the conservation law for the latent heat plays an important role. Indeed, for large undercoolings, we find evidence for a new steady-state finger, which we relate to local models $[3,4]$ of dendritic growth.

Our approach takes advantage of recent advances in computer power, as well as algorithmic development. We use a two-dimensional phase-field model of solidification [10-18], where a scalar field $\phi(\vec{x}, t)$ determines whether the phase at field point $\vec{x}$ and time $t$ is solid $(\phi=+1)$ or liquid $(\phi=-1)$. A useful approach introduced in Ref. [15] allows for the elimination of kinetic undercooling. We also use finite-element adaptive-grid refinement techniques, introduced by Provatas and collaborators [16], wherein a fine mesh is used near the rapidly varying solidmelt interface, and a coarse mesh is used in the bulk phases where quantities vary slowly with space and time. Let the dimensionless temperature be $\Theta=c_{p}\left(T-T_{M}\right) / \ell$, where $c_{p}$ is the specific heat at constant pressure, $T_{M}$ is the melting temperature, and $\ell$ is the latent heat of the fusion. Then the equations of motion are $[16,17]$

$$
\frac{\partial \Theta}{d t}=D \nabla^{2} \Theta+\frac{1}{2} \frac{\partial \phi}{\partial t},
$$

and

$$
\begin{aligned}
A^{2}(\vec{n}) \frac{\partial \phi}{d t}= & \vec{\nabla} \cdot\left[A^{2}(\vec{n}) \vec{\nabla} \phi\right]+\left[\phi-\gamma \Theta\left(1-\phi^{2}\right)\right]\left[1-\phi^{2}\right] \\
& +\frac{1}{2} \vec{\nabla} \cdot\left[|\nabla \phi|^{2} \frac{\partial A^{2}(\vec{n})}{\partial(\vec{\nabla} \phi)}\right],
\end{aligned}
$$

(C) 2001 The American Physical Society 
where $D=\mathcal{D} \tau_{0} / W_{0}^{2}$ and $\mathcal{D}$ is the thermal diffusivity. All quantities were measured in units of the microscopic time and length scales, $\tau_{0}$ and $W_{0}$. The parameter $\gamma$ is chosen to be $1.5957 D$ to eliminate kinetic undercooling [15], and thereby relate $\tau_{0}$ and $W_{0}$ to experimental values. Anisotropy enters through $A(\vec{n})$, where $\vec{n}=\vec{\nabla} \phi /|\nabla \phi|$ is the unit vector normal to the contours of $\phi: A(\vec{n})=[1-3 \epsilon]\left[1+\frac{4 \epsilon}{1-3 \epsilon}\left(\left(n_{x}\right)^{4}+\left(n_{y}\right)^{4}\right)\right]$, where $\epsilon$ is a constant [16]. We used $\epsilon=0.05$ [8,19]. The width of the interface is $W(\vec{n})=W_{0} A(\vec{n})$ and the characteristic time $\tau(\vec{n})=\tau_{0} A^{2}(\vec{n})$ [15]. By using $d_{0}=0.5539 / D$, the boundary condition at the interface [18] is $\Theta_{\text {int }}=-d(\vec{n}) K$, where $K$ is the interface curvature, and $d(\vec{n})=d_{0}\left[A(\vec{n})+\partial^{2} A / \partial\left(\cos ^{-1} n_{x}\right)^{2}\right]$ is the capillary length. The simulations were performed using Neumann boundary conditions: $\vec{n} \cdot \vec{\nabla} \phi=0$ and $\vec{n} \cdot \vec{\nabla} \Theta=0$, so that heat cannot leak out of the channel. Solidification is initiated by a small half disk of radius $R_{0}$ centered at the bottom of the channel. The interface is initially set to its equilibrium value $\phi(\vec{x})=-\tanh \left(x-R_{0}\right) / \sqrt{2}$. The initial temperature is $\Theta=0$ in the solid phase, which decays exponentially to its asymptotic value $\Theta=\Delta$ at $x \rightarrow \infty$ in the far field. The time step was chosen to be 0.016 , and the minimum grid size for the adaptive grid was 0.78 and 0.39 , for the low- and high- $P$ regimes, respectively.

With this approach, we can make a quantitative comparison to experiment as well as theory, since as $P \rightarrow 0$ these equations reduce to those of the anisotropic viscous finger. Motivated by the experiment of Molho et al. [8], the first set of simulations were done in a wide channel $L / d_{0}=121000$ with a rectangular cross section of $1600 W_{0}$ by $102400 W_{0}(319.3 \mu \mathrm{m} \times 2 \mathrm{~cm})$, similar to the experimental capillary cells [8] $(200 \mu \mathrm{m} \times 6 \mathrm{~cm})$. To examine the low Péclet regime, the simulations were done at low undercoolings $\Delta=0.01,0.05,0.1,0.125$, and 0.15 , with $D=40$ and $R_{0}=30$. After a short period of acceleration, tip velocity decreases to an asymptotic value, following approximately an inverse square root of time. To estimate this asymptotic tip velocity and width of the fingers, we extrapolated our transient results by using a scaling ansatz, as in Ref. [17], and by fitting to a highorder polynomial. The scaling ansatz is shown as an inset of Fig. 1; we scaled the width of the finger with $t^{\alpha}$, and the length of the finger with $t^{\beta}$, obtaining very good data collapse for the entire shape of the finger for different undercoolings [20].

This method and the polynomial fits gave consistent results for $\lambda$, which are shown in Fig. 1, where $V$ appears through the dimensionless surface tension $\kappa=\frac{D d_{0} \pi^{2}}{L^{2} V(1-\lambda)^{2}}$. We found that for these channel dimensions the dimensionless finger width, $\lambda$, is smaller than 0.5 , and decreases with increasing velocity, as predicted in the solvability theory of Kessler et al. [7]. Also shown are the results of the experiment and the solvability solution for the anisotropic

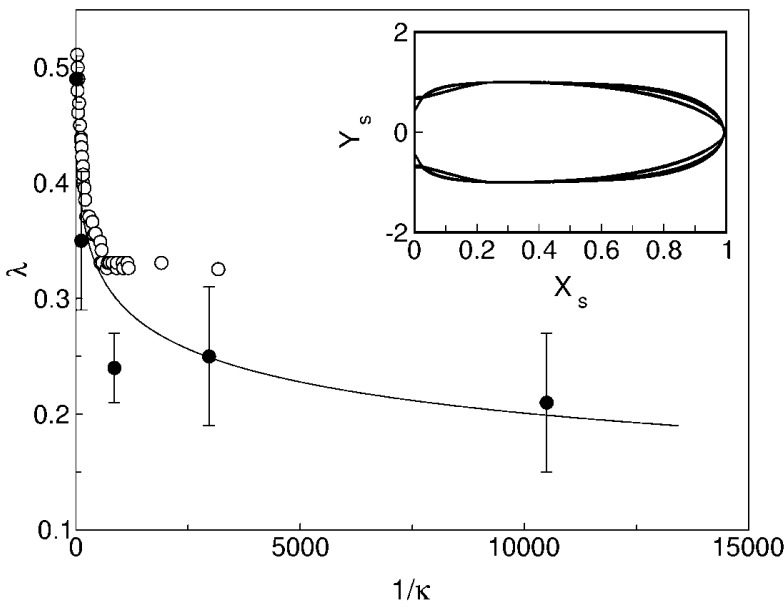

FIG. 1. Finger width $\lambda$ vs velocity $V$ for small Péclet numbers. Solvability theory $[7,21,22]$ (solid line, $\epsilon=0.05$ ), experimental results [8] (empty circles), and present simulations (filled circles, $\epsilon=0.05)$. Inset: scaling collapse used to estimate $V$ for $\Delta=$ 0.05 (thin line), and $\Delta=0.15$ (thick lines). $X_{s}$ is the scaled length of the finger, and $Y_{s}$ is the scaled width. For $\Delta=0.05$ all profiles collapse. For $\Delta=0.15$, seven profiles are shown, which collapse quickly for late times.

viscous finger [7,21,22]. With no adjustable parameters, our phase-field model results are consistent with the experimental observations $[8,19]$ and compare very well to the theoretical prediction $[7,21,22]$. Our results provide the first quantitative test of that prediction of solvability theory for anisotropic viscous fingers, confirming those predictions.

This correspondence between solidification in a channel and the anisotropic viscous finger, which we have exploited above, is restricted to small Péclet numbers, and to a transient time regime which diverges as $P \rightarrow 0$. For later times, and larger $P$, the situation is different: from thermodynamics, the only asymptotic solution for an arbitrarily long solid finger within a liquid channel is $\lambda=\Delta$, since $\Delta$ is the fractional amount of solid in equilibrium [7,23]. For long times, far beyond those investigated above, it is expected that the finger shape obtained above is unstable due to latent heat piling up in front of the finger. For example, Pelce [23] pointed out that the finger is unstable to a tip widening instability unless $d \lambda / d V>0$ : If $\lambda$ is a decreasing function of $V$, then a perturbation giving rise to a wider finger causes the finger to slow down, leaving more heat in the vicinity, causing it to slow more, and flatten until the finger reaches the size of the channel.

To investigate this regime, we simulated larger Péclet numbers in a narrow channel $L / d_{0}=355$, of dimension $100 W_{0}$ by $1600 W_{0}$. The simulations were performed at $\Delta=0.25-0.95$, with $D=2$ and $R_{0}=4$. Figure 2 shows the dependence of $V$ on time. There is a marked qualitative change at $\Delta \approx 0.5$. For $\Delta<0.5$, a tip widening instability takes place. If we estimate an effective $V$ and $\lambda$ from a transient regime before this instability takes place, we find 


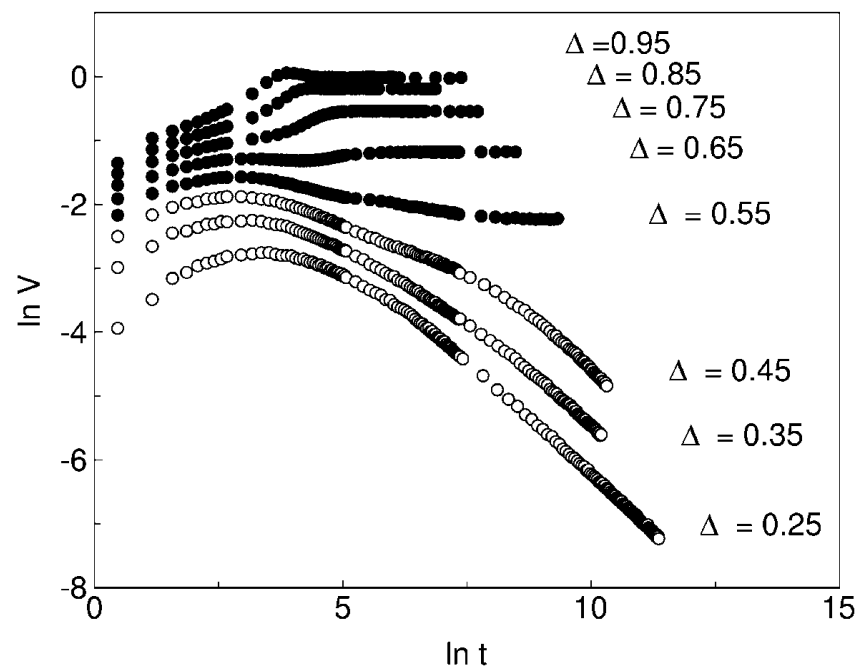

FIG. 2. Velocity $V$ vs time for various undercoolings $\Delta$, for larger Péclet numbers. Fingers with tip-widening instability $\Delta<0.5$ (open circles). Note qualitative change in velocities for stable fingers $\Delta>0.5$ (filled circles).

that $\lambda$ is a decreasing function of $V$, as would be expected: the dependence of $\lambda$ on undercooling for these fingers is shown by the open circles in Fig. 3; inset (a) of Fig. 3 shows one of these fingers while the instability is taking place.

In contrast, for $\Delta>0.5$, in the presence of anisotropic surface tension, the fingers rapidly achieve a well-defined constant velocity (closed circles in Fig. 2) and shape. Fits to $V$ give $P=5.4-49$. In Fig. 3, we show that their finger widths obey $\lambda=\Delta$; inset (b) shows one of these fingers. Hence these fingers satisfy thermodynamic and stability requirements, since $\Delta \propto V$. This new class of stable fingers becomes unstable to tip splitting if the surface tension is isotropic. We do not have an explanation for the apparent stability criterion $\lambda \geq 0.5$, although it is interesting to

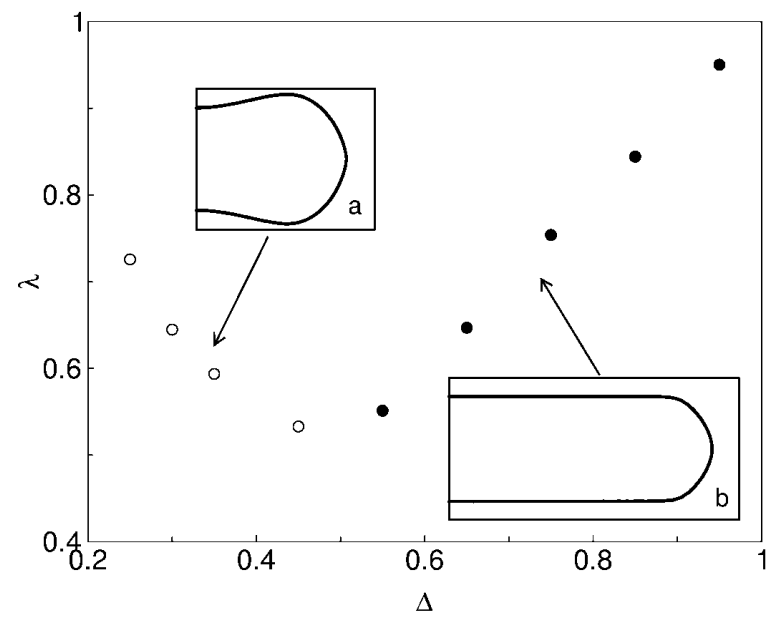

FIG. 3. Finger width $\lambda$ vs undercooling $\Delta$ for larger Péclet numbers. Fingers with tip-widening instability [open circles; inset (a)]. Stable fingers [filled circles; inset (b)]. note the reappearance of this bound for isotropic viscous fingers in the new context of rapidly growing anisotropic solid fingers. Nevertheless, for high undercoolings close to unity, we can suggest an origin for these new fingers.

For high undercoolings, the thermal boundary layer around the solidifying finger becomes small. As shown by Ben-Jacob et al. [4], in the limit $\Delta \rightarrow 1$ the interface velocity and curvature are determined locally, as in, e.g., the geometric [3] and boundary-layer [4] models of solidification. In such models, the steady-state shape is determined by the curvature $K$ as a function of only the normal velocity in the limit of vanishing surface tension. That is, for small $K$ and $n_{x}, K=f\left(n_{x}\right)=a n_{x}+b n_{x}^{3}+\cdots$. For the free boundary-layer model [4], the coefficient $a$ is exactly zero, and $b \propto(1-\Delta)$, giving rise to a parabolic shape. However, it is easy to show that a nonzero $a$ is required for a finger of finite width; here the value of $a$ is determined by the thermodynamic requirement $\lambda=\Delta$. This gives rise to the new steady-state solution:

$$
x=-B(\Delta / \pi) \ln \cos (\pi y / \Delta),
$$

where $1 / B=\sqrt{1+(b \Delta / 2 \pi)^{2}}-(b \Delta / 2 \pi)$. This form gives good one-parameter fits to the fingers, as shown in Fig. 4 (and fits which are significantly better than those from the shape $b \equiv 0$ ). Since the form is obtained from an expansion in $n_{x}$, it is a better fit in the tails than at the tips. Nevertheless, the fitted dependence of $b$ on $\Delta$ is reasonably well described by $b \propto(1-\Delta)$, for $\Delta \rightarrow 1$ as expected.

To conclude, we have investigated solidification in a channel in low and high undercooling regimes. Our results for low undercooling are consistent with earlier experimental work, and compare well to theory, providing the

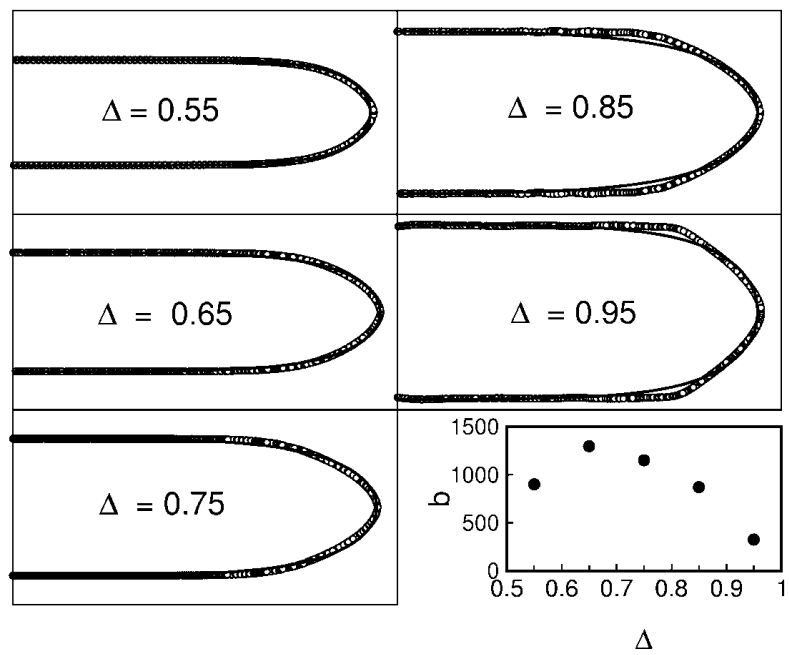

FIG. 4. Numerical (open circles) and analytical (lines) results for stable fingertip profiles, at undercoolings $\Delta=0.55-0.95$. Analytic fits, from an expansion valid in the tail, involve one parameter which should obey $b \propto(1-\Delta)$ for $\Delta \rightarrow 1$. $b$ vs $\Delta$ (bottom inset) shows reasonable agreement. 
first quantitative test of solvability theory for anisotropic viscous fingers. For large undercoolings, we find new phenomena, a solid finger which satisfies stability and thermodynamic criterion. We further argue for an analytic form of the shape, based on local models of solidification, which fits our results from numerical simulation.

This work was supported by the Natural Sciences and Engineering Research Council of Canada, le Fonds pour la Formation de Chercheurs et l'Aide à la Recherche du Québec. We thank Hong Guo, François Drolet, Mikko Haataja, and Martin Dubé for useful discussions.

[1] J. S. Langer, Rev. Mod. Phys. 52, 1 (1980).

[2] For reviews, see P. Pelce, Dynamics of Curved Fronts (Academic, London, 1988); B. Caroli, C. Caroli, and B. Roulet, in Solids Far From Equilibrium, edited by G. Godrèche (Cambridge University Press, Cambridge, 1992).

[3] R. C. Brower, D. A. Kessler, J. Koplik, and H. Levine, Phys. Rev. Lett. 51, 1111 (1983); Phys. Rev. A 29, 1335 (1984).

[4] E. Ben-Jacob, N.D. Goldenfeld, J.S. Langer, and G. Schön, Phys. Rev. Lett. 51, 1930 (1983); Phys. Rev. A 29, 330 (1984).

[5] B. Shraiman, Phys. Rev. Lett. 56, 2028 (1986); D. C. Hong and J. S. Langer, Phys. Rev. Lett. 56, 2032 (1986); R. Combescot, T. Dombre, V. Hakim, Y. Pomeau, and A. Pumir, Phys. Rev. Lett. 56, 2036 (1986).

[6] P. G. Saffman and G. I. Taylor, Proc. R. Soc. London A 245, 312 (1958).

[7] D. A. Kessler, J. Koplik, and H. Levine, Phys. Rev. A 34, 4980 (1986).

[8] P. Molho, A. J. Simon, and A. Libchaber, Phys. Rev. A 42, 904 (1990).

[9] E. Ben-Jacob, R. Godbey, N. D. Goldenfeld, J. Koplik, H. Levine, T. Mueller, and L. M. Sander, Phys. Rev. Lett. 55, 1315 (1985).

[10] A demonstration of a working phase-field method for dendritic growth is shown in R. Kobayashi, Physica (Amsterdam) 63D, 410 (1993).

[11] Early papers which argue for the phase-field method being applied to dendrites include J.S. Langer, Directions in Condensed Matter (World Scientific, Singapore, 1986), p. 164; G. Caginalp, Arch. Ration. Mech. Anal. 92, 205 (1986); Ann. Phys. (N.Y.) 172, 136 (1986); J. B. Collins and H. Levine, Phys. Rev. B 31, 6119 (1985); see also G. Caginalp and J. T. Lin, IMA J. Appl. Math. 39, 51
(1987); A. McNabb and L. Bass, IMA J. Appl. Math. 43, 1 (1989); G. Caginalp, Phys. Rev. A 39, 5887 (1989); G. Caginalp and E. A. Socolovsky, J. Comput. Phys. 95, 85 (1991).

[12] A. A. Wheeler, W. J. Boettinger, and G. B. McFadden, Phys. Rev. A 45, 7424 (1992); A. A. Wheeler, G. B. McFadden, and W. J. Boettinger, Proc. R. Soc. London A 452, 495 (1996); J. A. Warren and W. Boettinger, Acta Metall. Mater. A 43, 689 (1995).

[13] K. R. Elder, F. Drolet, J. M. Kosterlitz, and M. Grant, Phys. Rev. Lett. 72, 677 (1994); Phys. Rev. E 61, 6705 (2000).

[14] S.-L. Wang and R.F. Sekerka, Phys. Rev. E 53, 3760 (1996).

[15] A. Karma and W.-J. Rappel, Phys. Rev. E 53, 3017 (1995); 57, 4323 (1998).

[16] N. Provatas, N. Goldenfeld, and J. Dantzig, Phys. Rev. Lett. 80, 3308 (1998).

[17] N. Provatas, N. Goldenfeld, J. Dantzig, J. C. Lacombe, A. Lupulescu, M. B. Koss, M. E. Glicksman, and R. Almgren, Phys. Rev. Lett. 82, 4496 (2000).

[18] K. R. Elder, M. Grant, N. Provatas, and J. M. Kosterlitz give a discussion of the sharp-interface limit (to be published).

[19] We suspect that the remaining discrepancy with experiment, in comparison to the better agreement we obtain between solvability theory and our simulations, is due to our use of an anisotropy coefficient $\epsilon=0.05$. While this is the value given for pivalic acid in the experimental paper [8], later work gives $\epsilon=0.025$ [M. Muschol, D. Liu, and H.Z. Cummins, Phys. Rev. A 46, 1038 (1992)] which would result in closer agreement. See also M. E. Glicksman and N. B. Singh, J. Cryst. Growth 98, 277 (1989); further experiments determining surface tension anisotropy as well as kinetic undercooling would be useful.

[20] We find effective exponents $\alpha=0.29,0.47,0.54,0.61$, 0.73 and $\beta=0.23,0.27,0.23,0.17,0.05$ for $\Delta=0.01$, $0.05,0.1,0.15$. The values of the fits to $\beta$, in particular, are significantly below those for the free dendrite (Ref. [17]). Here they describe effectively the approach of the finger to the width of the channel in the $y$ direction.

[21] A. T. Dorsey and O. Martin, Phys. Rev. A 35, 3989 (1987).

[22] E. Corvera, H. Guo, and D. Jasnow, [Phys. Rev. E 52, 4063 (1995)] give the explicit analytic form for the anisotropic viscous finger.

[23] P. Pelce, Europhys. Lett. 7, 453 (1988); P. Pelce and A. Pumir, J. Cryst. Growth 73, 337 (1985); see also D. A. Kessler and H. Levine, Mod. Phys. Lett. B 8, 945 (1988); see also E. Brener, H. Müller-Krumbhaar, Y. Saito, and D. Temkin [Phys. Rev. E 47, 1151 (1993)] for a study of solidification in a wide channel. 